\title{
Bilingual and monolingual children attend to different cues when learning new words
}

\section{Chandra L. Brojde, Sabeen Ahmed and Eliana Colunga*}

Department of Psychology and Neuroscience, University of Colorado, Boulder, CO, USA

Edited by:

Catherine M. Sandhofer, University of California Los Angeles, USA

\section{Reviewed by:}

Teresa Mitchell, University of Massachusetts Medical School, USA Amina Abubakar, Tilburg University, Netherlands

\section{*Correspondence:}

Eliana Colunga, Department of Psychology and Neuroscience, 345 UCB, University of Colorado Boulder, Boulder, CO 80309-0345, USA e-mail: colunga@colorado.edu
The way in which children learn language can vary depending on their language environment. Previous work suggests that bilingual children may be more sensitive to pragmatic cues from a speaker when learning new words than monolingual children are. On the other hand, monolingual children may rely more heavily on object properties than bilingual children do. In this study we manipulate these two sources of information within the same paradigm, using eye gaze as a pragmatic cue and similarity along different dimensions as an object cue. In the crucial condition, object and pragmatic cues were inconsistent with each other. Our results showed that in this ambiguous condition monolingual children attend more to object property cues whereas bilingual children attend more to pragmatic cues. Control conditions showed that monolingual children were sensitive to eye gaze and bilingual children were sensitive to similarity by shape; it was only when the cues were inconsistent that children's preference for one or the other cue was apparent. Our results suggest that children learn to weigh different cues depending on their relative informativeness in their environment.

Keywords: bilingualism, word learning, language development

\section{INTRODUCTION}

What impact do different language environments have on the process of language acquisition? According to the Attentional Learning Account (Smith, 2000; Smith et al., 2010), as children learn words, they learn to tune attention efficiently, highlighting dimensions that are relevant for lexical categories in the particular language being learned, thus becoming better and more efficient word learners, optimized for learning the language that surrounds them. Evidence for this idea includes training studies (Samuelson, 2002; Smith et al., 2002), cross-linguistic studies (Yoshida and Smith, 2003; Colunga and Smith, 2005; Colunga et al., 2009), and computational modeling work (Samuelson, 2002; Colunga and Smith, 2005). In this paper we look at the way bilingual and monolingual environments shift the way in which children weigh different sources of information when learning new words.

Research shows that children attend to many different sources of information including pragmatic cues, social cues, and cues from the properties of objects themselves as ways to help them learn language and more specifically object names. Young children have been shown to attend to changing contexts, like the presence or absence of a speaker, when learning a new word (e.g., Akhtar et al., 1996; Diesendruck, 2005). They also attend to the properties of objects (e.g., shape, color, texture) to extend a word to other objects (e.g., Colunga and Smith, 2005). Finally, children attend flexibly to the linguistic context depending on whether an interlocutor is looking or pointing at an object when naming it (e.g., Baldwin, 1993; Grassmann and Tomasello, 2010). To what extent is this attentional shifting dependent of the child's environment? The evidence suggests that monolingual and bilingual children may attend to different sources of information when learning words.

\section{WORD LEARNING IN BILINGUAL AND MONOLINGUAL CHILDREN}

Monolingual and bilingual children's word learning environments differ in many ways. Aside from the obvious difference in the number of words to learn and the degree of exposure to each language, bilingual children are also faced with the additional task of discovering from which language each word comes. Additionally, because bilingual children are constantly faced with the possibility of others not knowing one of their spoken languages, they must continuously attend to whether a speaker knows two languages. This has led some researchers to suggest that bilingual children may have the need to attend more to the speaker as a cue for word meaning. Indeed, research suggests that from early on, bilingual children are sensitive to pragmatic information. For example, bilingual children can use information about whether an interlocutor knows both languages or not or whether a speaker was present in a room when a word was first introduced or not to help them determine the meaning of a word (Diesendruck, 2005). As we will review next, children who are growing up in either monolingual or bilingual environments attend to a variety of sources of information, but does the relative weight they place on these different sources dependent on the demands of their environment? In other words, are bilingual children more sensitive to pragmatic information than monolingual children? And, if bilingual children attend more to cues from the speaker, does that mean that they attend less to other sources of information? These are the questions we ask in this experiment.

When it comes to learning object names, the properties of the objects themselves become an important source of information. From about 2 years of age, children generalize new nouns in very systematic ways depending on their perceptual properties. For 
example, shown objects with features typical of animate beings, like eyes or legs, children generalize the name narrowly to things that are similar in multiple properties, especially shape and surface properties such as texture and/or material, such as furry or smooth (e.g., Yoshida and Smith, 2003). Given a solid thing with multiple parts and constructed shape (that is, an artifact), children generalize the name more broadly to all things that match the exemplar object in shape (e.g., Jones et al., 1991). Finally, given a non-solid substance, children extend the name by surface properties, especially material (e.g., Soja et al., 1991). That is, young children use information on whether the object to which a noun is applied is solid or not, and whether it has eyes or not, as a cue to what the extension of the noun should be.

Differences between how monolingual and bilingual children use object property cues may also exist due to the ways in which people in the children's environment teach them novel words. When parents teach their children a new word, parents highlight characteristics of an object in order to help the child extend that word to other perceptually similar objects (Merriman and Kutlesic, 1993). For instance, in teaching the word ball, a parent may draw attention to its roundness in order to help the child extend the word to other balls. In contrast, a bilingual parent might draw from the child's vocabulary on the other language when introducing a new word, for example, pointing out that the thing called ball is what the child knows as pelota.

There is relatively little research that directly looks at the question of how monolingual and bilingual children allocate attention during word learning, but the evidence suggests that, compared to bilingual children, monolingual children attend more to object properties when learning a new word (Merriman and Kutlesic, 1993; Haryu and Imai, 1999). Merriman and Kutlesic (1993) found that monolingual children, compared to bilingual children, are more likely to use the highlighted feature of an object in learning a novel noun as a necessary prerequisite to extend the noun to other things. Likewise, in the study done by Rosenblum and Pinker (1983), in which participants were asked to cite a reason to explain why they applied a novel label to one object, but not to another, monolingual children were inclined toward justifying their reasons based on the object's attributes, such as "because they're both green" or "because it has four legs" (Rosenblum and Pinker, 1983, p. 778). However, when bilingual children were asked to justify their reasoning behind extending the novel name to one object but not to another, they tended to cite reasons based on personal and social context, for instance, "because it's in our game" or "because you told me and I know. ..I know what it is and you know what it is." Thus, this study suggests that whereas monolingual children may assume nouns are inherent attributes of objects, bilingual children may be aware that names are subjective social conventions (Rosenblum and Pinker, 1983; Diesendruck, 2005).

The relative difference in how monolingual and bilingual children make use of social-pragmatic cues may arise between the two language groups as a result of their different linguistic environments. According to the socio-pragmatic account, children learn the meanings of words by using pragmatic cues the speaker provides, which could be through social discourse or behavioral gestures (e.g., Baldwin, 1993; Akhtar et al., 1996; Clark and Grossman, 1998; Jaswal and Hansen, 2006). A simple example of a social cue is demonstrated in the following: if a speaker says, "Henry is hungry. What does Henry want?" Between the choices of an apple and a pair of tongs, the child would choose the apple because the speaker indicated that Henry is hungry (Haryu and Imai, 2002). The idea is that, when learning a word, a child deduces its meaning from what he or she infers as the speaker's intended meaning.

One of the most widely studied pragmatic cues are behavioral cues, where the speaker would point to or look at an object. Previous research has found that young children attend to a speaker's eye gaze to determine a speaker's intended referent (Baldwin, 1993). Not only are children sensitive to gaze direction, but pointing can be used to refer to a new word as well. For example, when asked for the referent of a novel name, "Get the dax," while offered a choice between a familiar, known, object, and a novel object, children will choose a novel object over a familiar object as the referent for the novel noun (sometimes called "mutual exclusivity bias"). However, if the experimenter gives extra cues by pointing to the familiar object, this preference can be overridden, and children will choose the object the experimenter is pointing at even if they already have a name for it Grassmann and Tomasello (2010). Such receptiveness to pragmatic cues helps children make use of their environment during language acquisition.

In sum, monolingual and bilingual children grow up in linguistic environments that have different characteristics and present different learning demands. Previous research on children's learning of multiple labels for an object has shown that bilingual and monolingual children may have different expectations about how words map to meanings. Here we suggest that the demands on bilingual children may lead to an increased reliance on or awareness of socio-pragmatic cues. On the other hand, object properties may be highlighted more frequently in the experience of monolingual children. Evidence based on children's explanations of their own novel noun generalizations suggests that this is the case, but to our knowledge there has been no work looking at the relative reliance on these two sources of information, object properties and socio-pragmatic cues, within a single paradigm that directly tests children's novel noun generalizations. We address this in our study.

\section{THE CURRENT STUDY}

All children flexibly attend to linguistically relevant information as they learn novel words, whether that information is pragmatic in nature or related to the object properties of new objects. Does the attention afforded to these cues relative to one another depend on one's environment? One must bear in mind that both bilingual and monolingual children have the capacity to attend to both pragmatic cues and object properties when learning a new object name, the question is whether there are differences in the degree of attention paid to the two types of cues between the two language groups. Whereas prior research has found that monolingual children attend more to object properties compared to bilingual children, no previous research has pitted the two types of cues against one another by looking at the relative differences between bilingual or monolingual children's attention to the two types of cues.

In order to directly examine the differences in attention to pragmatic and object cues between monolingual and bilingual children, we tested whether the amount of attention devoted to 
the two types of cues would differ when they were in conflict, each cue suggesting a different correct answer. The cues must be provided simultaneously in order to test this comparison explicitly. To do this, we asked monolingual and bilingual children to generalize the name of a novel object to other objects that matched in different properties. The objects were grouped in a way that would cue participants to a highlighted object property. The experimenter looked toward or away from these objects, thereby providing a congruent or incongruent pragmatic cue. Additionally, children's preference for objects when they were just given object property cues (i.e., experimenter looks straight at the child) or just given pragmatic cues (i.e., property of an object is not highlighted, experimenter looks at objects) was also measured to provide a baseline on the relative difference in attention given to each by monolingual and bilingual children.

All objects were solid artifacts, so in the absence of any other cues, we would expect children to generalize by shape. However, we predicted that the different extra cues would guide bilingual and monolingual children's generalizations in different ways. First, the predictions in the single-cue control conditions are clear: both bilingual and monolingual children should be able to use the cue offered, whether it is eye gaze or grouping objects by their physical properties. Similarly, when both cues are congruent, both language groups should be able to use them to generalize the novel noun to new objects correctly, in the way suggested by both cues. The critical condition is the incongruent cue condition. If bilingual children and monolingual children rely on different sources of information to different extents, we predict that performance in the incongruent condition will diverge between the two groups. More specifically, if bilingual children place higher importance on pragmatic cues than monolingual children, their performance in the incongruent condition should be the same as in the pragmatic only condition. In contrast, monolingual children's performance in the incongruent condition should be the same as their performance in the object cue only condition.

\section{EXPERIMENT 1 \\ MATERIAL AND METHOD \\ Participants}

A total of 16 monolingual (seven males, nine females) and 16 bilingual (seven males, nine females) children participated in the study. Bilingual children spoke English and at least one other language. As their second language, four children spoke French, two spoke Portuguese, two spoke Hindi, and one child each spoke Russian, German, Farsi, Korean, Spanish, Afrikaans, Bambara, and American Sign Language. The monolingual participants ( $M_{\text {age }}=30.26$ months $)$ were age-matched within \pm 7 weeks, to the bilingual participants $\left(M_{\text {age }}=29.87\right.$ months $)$, and the ages ranged from approximately 24-36 months. Participants were recruited by phone or email through a database listing children whose parents had voluntarily provided their information for the general use of the research lab's studies. Informed consent was obtained from the child's parent or guardian. All procedures were approved by the Institutional Review Board at the University of Colorado Boulder.

Demographically, both groups were similar. Seventy-five of the monolingual participants were white $(n=12), 18.7 \%$ were Asian $(n=3)$, and one monolingual participant was Hispanic. On the other hand, $62.5 \%$ of the bilingual participants were white $(n=10), 25 \%$ were Asian $(n=4)$, and one participant each was African-American and Hispanic. All monolingual and bilingual participants had at least one parent who attained graduate education beyond a 4-year undergraduate degree. Thus, all subjects came from professional, middle-class families, and there was no difference between the two groups in terms of socioeconomic status. All participants were compensated $\$ 5$ and were given a book at the end of the study.

The parents of the bilingual participants were asked to fill out a questionnaire regarding the extent of their child's bilingualism. This questionnaire asked the parents to report the degree to which their children understood and spoke both English and the other language on a scale from one to five [i.e., "Please describe the level of fluency for English and other languages that your child speaks (where 1 represents poor/no fluency and 5 represents perfect fluency for the child's age")]. Parents were asked to report the level of fluency for understanding each language separately.

An absolute difference between the score for understanding English and understanding the second language was calculated. A similar score was also calculated for children's ability to speak English and their second language. Only children with a difference of 3 or lower were classified as "bilingual" and were used in the study. For example, if a parent indicated a fluency of 1 for English, and a fluency of 5 for the second language, the respective score was 4 . This hypothetical participant would be classified as monolingual and would not be used in the study. However, if the fluency score for English was 3, and the fluency score for French was 3 , the corresponding score would be 0 ; this would indicate equal bilingualism, and the participant would be classified as bilingual. This criteria ensured that children were balanced bilinguals. Parents reported that on average, the difference in fluency of speaking the two languages $(M=2.06, \mathrm{SD}=1.00)$ was higher than the difference in understanding them $(M=1.27, \mathrm{SD}=1.16)$. Most children (11 out of 16) were judged by their parent to be more proficient in English than in their other language. Only a quarter of the children (4 out of 16) were judged by their parents to be more proficient in their other language than in English. In fact, ratings were overall higher in English than in their other language for speaking $(M=3.75$ for English vs. $M=2.43$ for Other, $t(15)=2.73, p<0.02$, two-tailed). Although children were also overall judged to be significantly more proficient at understanding English than at understanding their other language $(M=4.25$ for English and $M=3.5$ for Other), this difference was only marginally significantly $[t(15)=1.96, p=0.068]$. Because all procedures were conducted in English, this means only four children were tested in what their parents judged to be their non-dominant language.

\section{Materials}

There was a warm-up set made out of objects that were well-known to children. Three dogs made of three different materials were used (plush, rubber, and plastic), as well as a toy bird, a truck, and a seahorse. Dogs were used because most children at 30 months have little to no difficulty in recognizing them (Dale and Fenson, 1996). Approximately $97.1 \%$ of children in this age group know the word "dog." 
The experimental conditions used a total of 36 novel items created for this experiment, in four sets of nine each. In each set of nine, there was an exemplar object with a novel name. The novel names used for the four sets were zuly, flone, deej, and tizo. The novel names were randomly applied to the four exemplars from the four different sets. Four of the objects in each set matched the target in shape. Of these four shape-match objects, two matched only in shape, while the third object matched in both shape and color and the fourth object matched in both shape and texture. The other four objects in each set did not match the target object in shape, but matched in other dimensions. Two of these objects matched the target object in color and texture, while one matched the target object only in color, and the other matched the target object only in texture. Overall, in each set, four objects matched the target in shape, four objects matched in color, and four objects matched in texture. See Figure 1 for an example of the stimuli used in the study.

\section{Procedure}

All sessions were videotaped with the consent of the participants' parents. During the experiment, the child sat next to his or her parent, with the experimenter directly across the table, in order for the child to be fully aware of the direction of the experimenter's eye gaze. The child sat approximately two feet away from the experimenter. Before the experimental phase, there was a warm-up phase.

Warm-up phase. The purpose of the warm-up phase was to allow the children to get a sense of the task. In this phase, the participants were shown the three different dogs, the bird, the truck, and the seahorse all placed randomly on a tray. The experimenter first asked the participants "Can you find a dog?" Children received feedback during this phase. They were told that they were correct if they chose a dog and if they chose another object, they were told that it was not (e.g., "No, that is not a dog.") Once one dog had been identified the child was asked "Can you find another dog?" This procedure was repeated until all three dogs were chosen. The experimenter then asked the participants once more to

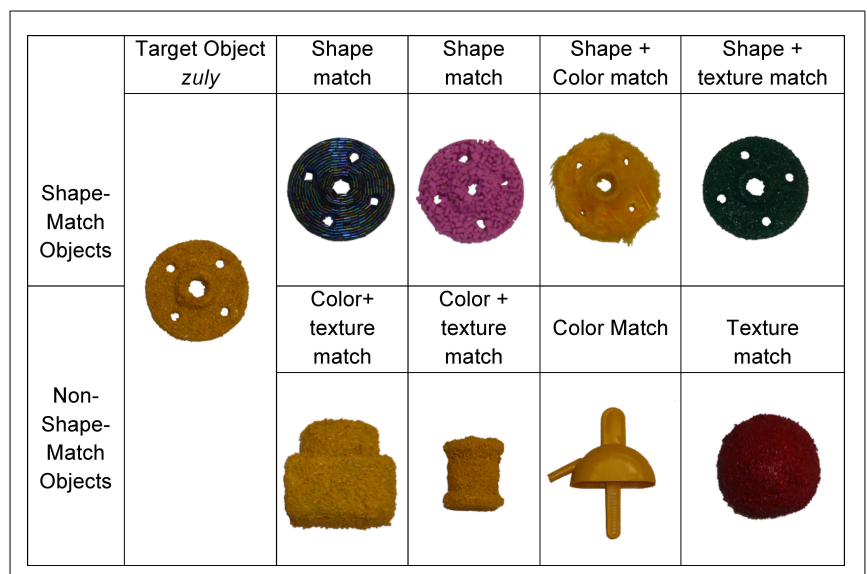

FIGURE 1 | One of the sets of stimuli used in Experiment 1 and Experiment 2. All sets had the same similarity structure. "find another dog," with the intention of the child indicating that there were no more dogs or to correct the child if he or she handed the experimenter another object on the tray. The purpose of this phase was to illustrate to the children that more than one object, but not all of the objects, on the tray were included in the requested category.

Experimental phase. During the experimental phase, children were given the name of a novel exemplar and asked to generalize the name to other novel objects matching in different properties under different cue conditions. The different cue conditions were run in the following order: pragmatic cue only, congruent, incongruent, object cue only. This was done to control for possible carry-over effects.

For each of the four conditions, the experimenter first presented the target object of a set placed in the center of a tray among the other objects in the same set, placed randomly about the target object. The experimenter named the target object with a novel name (e.g. "See the $z u l y$ "), repeating the novel label at least three times (e.g., "Look at/See/That's the zuly"). After the participant became familiar with the target object, it was removed from the tray, and the experimenter then quickly situated the remaining objects in different combinations and on different sides, depending on the conditions, which will be discussed later. The eight matching objects from the same set were then presented to each child on the tray.

After showing the child the exemplar, the experimenter asked the participant to retrieve an object with the novel label [e.g., "Can you find a $z$ uly?" "Do you see a(nother) zuly here?"] until the had chosen all eight objects or responded no, mirroring the process in the warm-up phase but without giving feedback.

The four conditions varied in two ways - how the objects were grouped on each side of the tray (object property cue) and where the experimenter was looking (pragmatic cue). See Figure 2 for an example of how the shape-match and non-shape-match objects were grouped, as well as the various directions of the experimenter's eye gaze. The children were tested to see if more attention was given to object property cues or to pragmatic cues in two

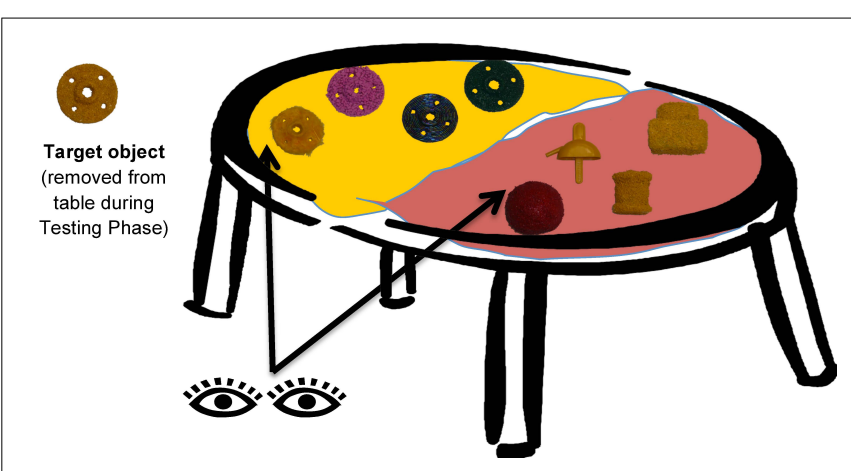

FIGURE 2 | In the conditions with object property cues, the test objects were grouped as shown; all shape matches on one side, all other items on the other. In the congruent condition the experimenter's eye gaze was directed to the shape-matching group, whereas in the incongruent condition the experimenter's eye gaze was directed to the opposite side of the table. 
conditions of interest: congruent and incongruent. In the congruent and incongruent conditions, all four shape matches were on one side of the tray and the non-shape matches were on the other side of the tray. This highlighted shape as an object property cue. In these conditions, pragmatic cues were also important. In the congruent condition, the experimenter continuously looked at the shape-match objects while requesting the novel object until the child made a selection, whereas in the incongruent condition, the experimenter constantly looked toward the non-shapematch objects instead, creating conflicting pragmatic and object property cues.

In the pragmatic cue only control condition, two objects matching the target object in shape and two objects that differed from the object in shape were placed on each side of a tray and the experimenter continuously looked toward one side when asking for the novel object, until the child chose an object. The objects were grouped in this manner so that the same number of shape-, color-, and texture matches were on each side of the tray. This eliminated the object property cue, because no one property (i.e., shape, color, texture) was explicitly highlighted.

Finally, an object cue only condition was also run as a control. As in the congruent and incongruent conditions, all four objects that matched the training object in shape were on one side, while the non-shape matches were on the other. The experimenter looked straight at the participant throughout this condition while asking for the novel object, thus eliminating the pragmatic cue.

Each participant completed all four conditions and saw four sets of novel objects in addition to the initial warm-up set. Throughout the experiment, the side of the tray on which objects were placed (i.e., the right and left of tray) was random. The novel names for each object and the order in which the sets were presented were counterbalanced across conditions. To equalize any carry-over effects from one condition to the next, all participants were tested with the four conditions in the same order: pragmatic cue only, congruent, incongruent, object cue only.

Coding. Children's choices of objects on both sides of the tray were calculated using the number of "incorrect" choices subtracted from the number of "correct" choices. In three of the conditions, congruent, incongruent, and object cue only, "correct" choices denoted the number of shape-match objects. Thus, for these conditions, the number of non-shape objects chosen was subtracted from the number of shape objects chosen. For the pragmatic cue only condition, "correct" choices denoted the number of objects chosen from the side the experimenter was looking at, minus the number of objects chosen from the opposite side, the "incorrect choices." This was done because in this condition, objects were not grouped by shape. Even if the participants chose more than four of the eight total objects, only the first four choices of objects were used for scoring with the purpose of including the order in which objects were chosen in the measure. Thus, a participant's score on this measure could range from -4 , where the child chose all four "incorrect" choices first, to 4, where the child chose all four "correct" choices first. For instance, if in the congruent, incongruent, and object cue only conditions, the child chose all four non-shape matches first, their score would equal -4 , whereas if the child chose all four shape objects first, the score would equal 4. In the pragmatic cue only condition, if the experimenter looked at the left side while asking for the novel object and the participant chose all four objects on the left side, the score would equal 4.

\section{RESULTS}

A 2 (cue congruency: congruent, incongruent) $\times 2$ (language group: bilingual, monolingual) mixed-factorial ANOVA was conducted with cue congruency as a within-subjects factor and language group as a between-subjects factor (see Figure 3 ). The mixed-factorial ANOVA used the dependent variable described above in which the number of non-shape matches was subtracted from the number of shape matches chosen by each participant. Overall, data analysis did not show a main effect for cue congruency, such that children chose the shape matches equally often in the congruent and incongruent conditions $F(1,30)=0.36, \eta^{2}=0.01$, n.s. However, there was a significant interaction between congruency and language group, $F(1,30)=4.08, \eta^{2}=0.12, p=0.05$., indicating that bilingual and monolingual children performed differently in the congruent and the incongruent conditions. Post hoc tests (all two-tailed) confirmed that bilingual children chose the shape-match objects marginally more in the congruent conditions than incongruent conditions, $t(15)=1.73, p=0.10$, whereas monolingual children, on the other hand, showed no difference across the two trials, $t(15)=1.09$, n.s. Finally, there was a significant main effect for language group, such that overall monolingual children chose the shape-match objects more often than the bilingual children, $F(1,30)=4.26, \eta^{2}=0.13, p<0.05$.

Comparing the two language groups in the incongruent trials was especially important, as the main question was to determine if children would pay more attention to pragmatic cues or to object property cues. Results from a planned comparison $t$-test showed that monolingual children noticeably paid more attention to object property cues by choosing more shape-match objects than did the bilingual children in this condition, $t(30)=3.07$, $p<0.01$. When the pragmatic cues and object property cues were congruent, monolingual, and bilingual children did not differ in the degree to which they chose the shape-match objects, $t(30)=0.27$, n.s.

To see what children would do if only one of the cues was available (i.e., either pragmatic cue or object cue), the two control conditions were analyzed separately. When children received only the pragmatic cue, both monolingual and bilingual children chose the objects at chance levels, $p$ 's $>0.05$, suggesting that neither group attended significantly to the pragmatic cues when this was the only cue available. Overall, in the object cue only condition, children performed similar to the congruent condition (i.e., objects grouped by shape but experimenter looks at the child). There was no difference in the children's choices of shape-match objects in this condition, $t(30)=0.27$, n.s, nor did these conditions differ from either the congruent or incongruent condition together or for each language group separately, all p's $>0.05$.

A one-sample $t$-test was run to compare each of the four conditions to chance for both language groups separately (see Table 1). 


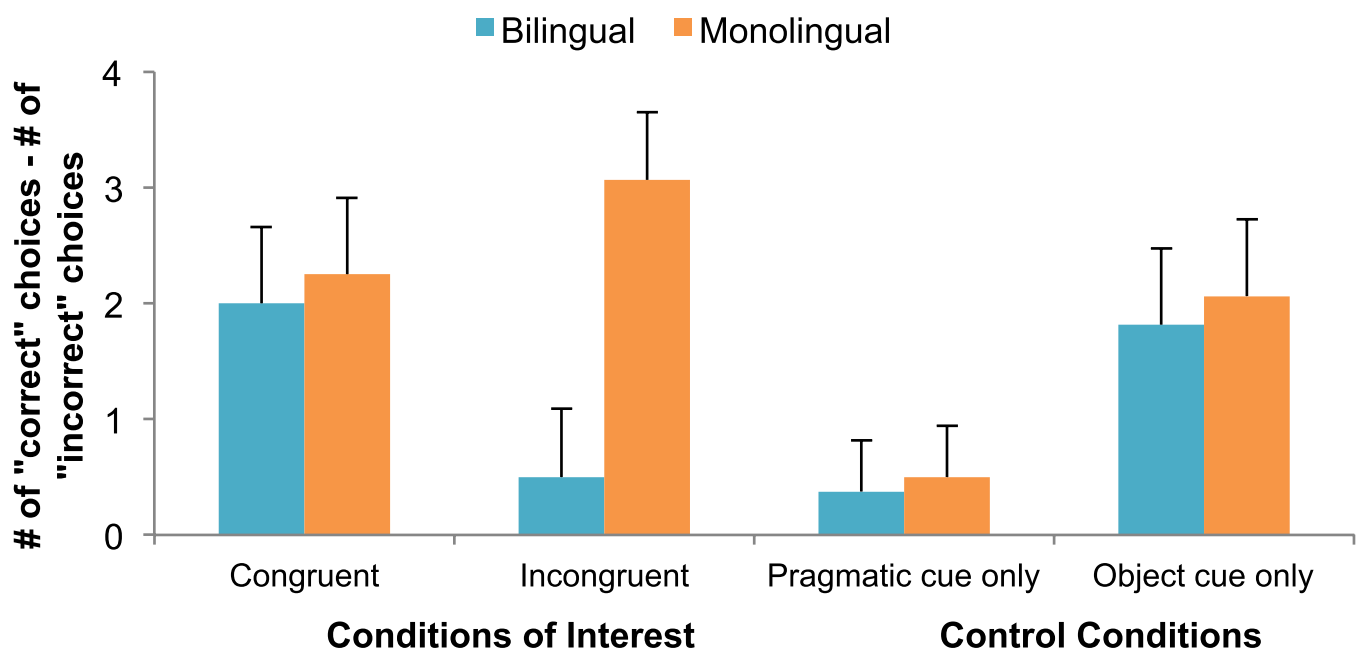

FIGURE 3 | Results of Experiment 1.

Table 1 | Results for conditions compared to chance for Experiment 1.

\begin{tabular}{lll}
\hline & \multicolumn{2}{c}{ Language group } \\
\cline { 2 - 3 } & Bilingual & Monolingual \\
\hline CONDITION & & \\
Congruent & $2.00^{*}$ & $2.25^{* *}$ \\
Incongruent & 0.50 & $3.06^{* *}$ \\
Pragmatic only & 0.38 & 0.50 \\
Object cue only & $1.81^{*}$ & $2.06^{* *}$ \\
\hline${ }^{* *} p<0.01,{ }^{*} p<0.05$. & &
\end{tabular}

In this study, since a child received a score ranging from -4 to 4 , a score of zero would indicate chance, meaning that the children showed no bias to choose either the shape-match or non-shapematch objects. A score above zero alternatively, would indicate a shape bias, since the participant picked more shape-match objects than non-shape objects. Overall, monolinguals chose shape-match objects relatively more than chance in three of the four conditions including the congruent, $t(15)=3.61, p<0.01$, incongruent, $t(15)=8.03, p<0.01$, and object cue only, $t(15)=4.12, p=0.001$, conditions. However, in the pragmatic cue only condition, monolingual children chose the shape-match objects equally as often as the non-shape-match objects, $t(15)=3.12, p<0.01$, showing that the pragmatic cue did have the effect of disrupting the expected shape bias.

Bilingual children showed a different pattern, however. In the congruent and the object cue only conditions, like monolingual children, bilingual children choose the shape-match objects more than expected by chance, $t(15)=2.83, p<0.05$ and $t(15)=2.75$, $p<0.05$, respectively. Similarly, in the pragmatic cue only condition bilingual children chose neither the shape or non-shapematch objects more, $t(15)=1.45, p=$ n.s. However, in the critical incongruent condition, children in the bilingual group behaved differently than monolingual children. When object property and pragmatic cues conflicted, bilingual children did not show a shape bias; instead, they chose both the shape and non-shape-match objects equally, $t(15)=0.67, p=$ n.s., as in the pragmatic only condition. This suggests that bilingual children paid relatively more attention to the pragmatic cues and thus ceased to show a shape bias when the cues were incongruent.

A series of Pearson's correlations were conducted for the bilingual group between the average number of shape matches chosen minus the number of non-shape matches chosen and the difference in understanding and speaking their two languages. This was done separately for each of the four conditions. No significant correlations were found between any of the conditions, all $p$ 's $>0.05$.

\section{DISCUSSION}

Overall, both monolingual and bilingual children attend to the object property cues when it was the only cue available or when both cues were available and were congruent. Yet when both the object property and pragmatic cues were available but incongruent, bilingual children comparatively attended less to the object property cue, suggesting some attention was diverted to the pragmatic cue. Monolingual children in comparison paid extensive to the object property cue in all conditions. In sum, these results suggest that bilingual children are more likely to attend to pragmatic cues than monolingual children when the two cues conflict.

There is another intriguing possibility, though. Recent evidence suggests that bilingual children may show more cognitive flexibility than monolingual children (e.g., Bialystok et al., 2010). For example, compared to monolingual children, bilingual children can succeed at a younger age in a dimensional change card sorting task in which they are asked to sort by one dimension (say color) and then switch to sorting by a different dimension (for example shape; Bialystok and Martin, 2004). Note that in trying to control for carry-over effects, we ran the conditions always in the same order: Pragmatic cue only, congruent, incongruent, and object cue only. Monolingual children, then, showed no preference for shape 
in the first condition, then showed a shape bias in the congruent condition in which every cue pointed to shape matches as the correct response, and then consistently generalized novel names by shape in each of the two following conditions, incongruent and object cue only. Bilingual children, in contrast, showed a shape bias only in the second and the fourth conditions. It is possible that because of their greater cognitive flexibility, bilingual children were able to switch from attending to shape, correctly, in the congruent condition, to not attending to shape in the incongruent condition, whereas monolingual children were stuck attending to shape once they had done it consistently and correctly in the congruent condition.

To account for this possibility, we ran a follow-up experiment with monolingual children reversing the order of the congruent and incongruent conditions. If monolingual children's preference for shape matches in the incongruent condition was due to carryover from the congruent condition, this preference should go away when the order of the congruent and incongruent conditions is reversed. However, if this is not the case, and monolingual's preference for shape matches in the incongruent condition indicates a preference for object property cues over eye gaze information, then monolingual children in Experiment 2 should show exactly the same pattern of performance as the monolingual group in Experiment 1.

\section{EXPERIMENT 2 MATERIAL AND METHODS \\ Participants}

A total of 10 monolingual children (four males, six females) participated in the study. The ages ranged from 26 to 36 months and $M_{\text {age }}=31.58$. Participants were recruited in the same manner as that of Experiment 1. The 16 monolingual children who participated in Experiment 1 were used for comparison. All monolingual participants recruited for Experiment 2 were Caucasian. All participants had at least one parent who attained graduate education beyond a 4-year undergraduate degree. Thus, all children came from professional, middle-class families so there was no difference in socioeconomic status between this group of monolinguals and the group of monolinguals from Experiment 1. All participants were compensated $\$ 5$ and were given a book at the end of the study.

\section{Materials}

The same materials as in Experiment 1 were used in this study, with the exception of the bilingual survey, as this experiment only used monolingual children.

\section{Procedure}

The procedure was the same as in Experiment 1, with the exception of the order in which the different cue conditions were run. The incongruent condition was run before the congruent condition. Thus, the conditions were run in the following order for all participants in Experiment 2: pragmatic cue only, incongruent, congruent, and object cue only. The incongruent condition was run before the congruent condition to test the hypothesis that in Experiment 2 monolingual children had failed to show sensitivity to pragmatic cues in the incongruent condition because they were "stuck" generalizing by shape after doing so, correctly, for the congruent condition.

\section{RESULTS AND DISCUSSION}

Once again, participants' choices of objects on both the left and right side of the tray were calculated by computing a score where the number of non-shape objects chosen was subtracted from the number of shape objects chosen. A 2 (cue congruency: congruent, incongruent $) \times 2$ (experiment: monolingual participants from Experiment 1, participants from Experiment 2) mixed-factorial ANOVA was conducted with cue congruency as a within-subjects factor and experiment as a between-subjects factor. There was no main effect of cue congruency, such that monolingual participants from Experiment 1 and monolingual participants from Experiment 2 chose the shape-match objects equally, $F(1,24)=1.54$, $p=$ n.s. Additionally, the interaction between cue congruency and experiment was not significant, $F(1,24)=0.03, p=$ n.s (see Figure 4). Thus, monolingual children from Experiment 1 and monolingual children from Experiment 2 chose shape-match objects on average equally in both experiments, even when the order of the congruent and incongruent conditions was switched (i.e., incongruent condition run first), indicating there were no order effects in the study.

Since there were no differences in the choices of monolingual participants between the two experiments, the finding that monolingual children attend more to object property cues is further supported. Monolingual children chose shape-match objects equally when the incongruent condition was run before the congruent condition, and when the congruent condition was run first. Thus, their performance in the incongruent condition in Experiment 1 was not due to an inability to stop attending to shape after the congruent condition. It is unlikely that the pattern of results was due to order effects from the order in which the conditions were run.

\section{GENERAL DISCUSSION}

In the current study, we investigated the consequences of linguistic environment on word learning. In particular, we tested how a bilingual environment might influence the way children attend to different sources of information in the process of language acquisition. Children learn words through a variety of means, by

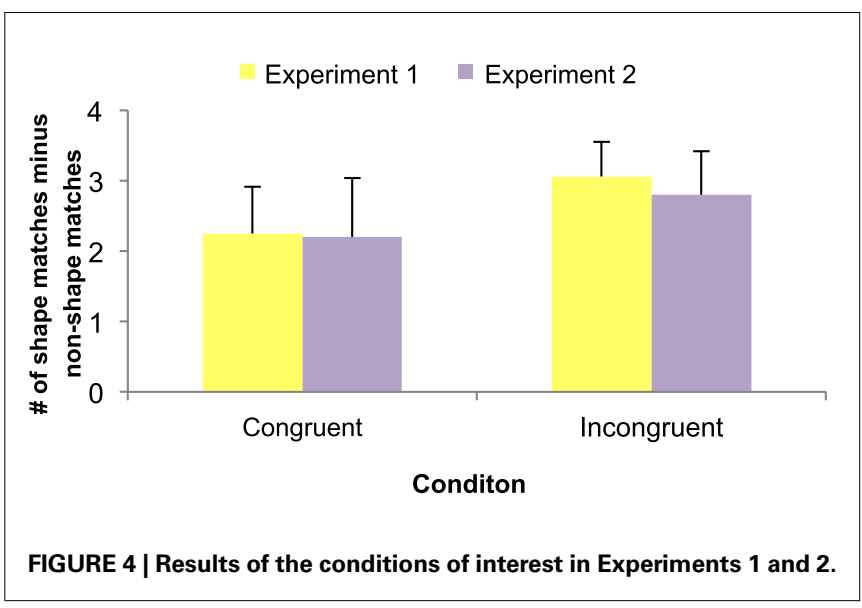


attending to the many cues provided by the caregivers in their environment. The type of environment to which children are exposed may have a significant impact in the way children learn words. Different environments may ultimately lead children to attend to particular linguistic cues more than others, depending on whether certain types of cues are provided to a greater extent or are more predictive of success in learning words in that environment. For instance, if a child learns that attending to the properties of an object helps him or her learn words at a faster rate, he or she may come to rely more on object property cues. On the other hand, if a child discovers that pragmatic cues from a speaker aided more in acquiring language, he or she may attend more to this type of cue when learning new words.

\section{SUMMARY OF RESULTS}

In this study, we compared the difference in attention to pragmatic and object cues among monolingual and bilingual 30-month olds. We tested whether bilingual and monolingual children differed in the extent to which they attended to pragmatic and object cues, particularly when the two cues were incongruent. Participants were asked to identify objects given novel names by the experimenter. Objects were grouped in a manner to draw the children's attention to a major property of the object; in this case, shape. During the study, the experimenter simultaneously provided pragmatic cues (e.g., looking toward these objects or away from these objects). Data were analyzed to determine which type of cue bilingual and monolingual children attended to a greater degree when the pragmatic and object property cues were incongruent.

Overall, monolingual children chose more objects matching in shape, and they chose shape-match objects even when the experimenter looked away from the shape-match objects while asking children to extend the novel label they learned in training (e.g., zuly). Furthermore, they chose shape-match objects more than chance in three of the four conditions - congruent, incongruent and object cue only, indicating a strong shape bias regardless of pragmatic cue. This result is in line with previous research indicating that by 2 years of age children systematically generalize novel nouns for solid artifacts by shape, ignoring differences in other dimensions such as color and texture (Landau et al., 1988; Smith et al., 2002; Colunga and Smith, 2005). We will discuss the possible reasons why monolinguals chose neither shape-match nor non-shape-match objects in the pragmatic cue only condition later.

On the other hand, bilingual children showed a shape bias only when shape was highlighted by grouping and the experimenter did not offer conflicting cues through their eye gaze but either looked neutrally at the child (object property cue condition) or looked at the group of objects matching in shape (congruent condition). In both of these conditions, bilingual children chose the shape-match objects more than chance. These results are in line with our predictions, as we expected bilingual children to exhibit a shape bias in these trials. Also in line with our predictions, bilingual children performed differently when the experimenter gave conflicting cues, choosing both the shape-match and non-shapematch objects equally. This pattern of results suggests that bilingual children attended to the experimenter's eye gaze, instead of using the highlighted feature of shape, and thus stopped showing a shape bias when the cues were incongruent.

How did the performance of the two groups compare relative to each other? When there was no conflict in what the different cues indicated, both language groups behaved the same way: First, in the object property cue, when the experimenter looked straight at the child while asking which other objects could be called by the novel noun out of a setting in which same-shaped objects were grouped together, both monolingual and bilingual children systematically generalized by shape. Second, in the pragmatic object cue, when the items were not grouped by their properties and the experimenter looked at one side of the table, neither group generalized by shape or by side of the sable. Finally, when the object property and pragmatic cues were congruent with the experimenter looking at a group of objects similar in shape, both monolingual and bilingual children generalized the novel noun by shape, as in the object property condition. This could mean that the highlighting of the shape dimension alone was enough to lead them to shape, as adding the eye gaze hint did not increase the likelihood of children choosing shape over what grouping same-shaped objects alone did. Interestingly, in the incongruent condition, when the cues conflicted, and the experimenter looked toward the group of non-shapematch objects while requesting the novel object, monolingual and bilingual children generalized the novel noun in different ways. Monolingual children generalized the novel noun as in the object property cue condition, choosing the shape-matched objects more than chance, and significantly more often than bilingual children. However, bilingual children in the incongruent condition did the same as they did in the pragmatic cue condition, choosing shape-match and non-shape objects equally and failing to show a shape bias. This suggests that bilingual children paid relatively more attention to the experimenter's eye gaze than monolingual children, at least when the cues were incongruent.

One curious finding is that when the experimenter only provided the pragmatic cue, neither monolingual children nor bilingual children extend novel nouns systematically choosing by shape or by the side to which eye gaze was directed. This may be seen as contradicting previous research (e.g., Baldwin, 1993). Our finding suggests that neither group afforded overwhelming attention to eye gaze. One difference between our experiment and previous work looking at eye gaze is the number of items from which the children had to make choices. We set out eight choices whereas previous work had used fewer, mostly just two items. It is possible that interpreting the eye gaze information becomes difficult when the visual field contains so many items. The very fact that in this condition (as in the incongruent condition), the items were not grouped by their perceptual properties might have made this more difficult to interpret eye gaze. Recall that in the conditions in which shape was not highlighted by grouping, equivalent matches of features were presented on each side of the tray: two shape matches and two color matches on one side, and two shape matches and two texture matches on the other side. This particular assortment of objects may have overwhelmed the participants, whereas grouping, on the other hand, might have allowed children to "unitize" all the alike objects and treat them as two groups instead of as eight objects. Although both bilingual and monolingual children failed to show evidence of following the pragmatic cue in the pragmatic 
cue only condition, our results suggest that bilingual children did attend to these cues when they conflicted with object property cues in the incongruent condition.

\section{FLEXIBLE ATTENTION IN DIFFERENT LINGUISTIC ENVIRONMENTS}

In general, it appears that, as hypothesized, bilingual children attend to pragmatic and object property cues differently relative to monolingual children; specifically, they appear to pay more attention to pragmatic cues than object property cues when both types of cues are available and in conflict. Although bilingual children performed similarly in the pragmatic cue and in the incongruent conditions, suggesting that they were indeed using eye gaze information, the fact that they did not systematically choose mismatched objects of the side the experimenter looked makes it hard to make any strong claims. That is, the effect of eye gaze in bilingual children is observed as a disruption of the shape bias we assume they would otherwise show, and that monolingual children do show. This raises the possibility, then, that bilingual children are just not understanding the task and thus showing no systematic choices. This interpretation is unlikely because bilingual children did show clear preferences in the two conditions in which objects were grouped by shape. Furthermore, bilingual children were no more likely to require corrective feedback during the warm-up trial than monolingual children. All this suggests that bilingual children were capable of following the directions of the task at hand. Moreover, no significant correlations were found between fluency in understanding and speaking both languages and performance in conditions. This brings us to the question of why do monolingual and bilingual children differ in the importance they give to pragmatic and object property cues when the two cues conflict? How do the linguistic environments of monolingual and bilingual children differ in a way that ultimately leads to the relative differences in attention afforded to object property and pragmatic cues?

Although bilingual and monolingual tend to hit developmental milestones in language acquisition at around the same time (Petitto et al., 2001), bilingual children's vocabulary may be significantly smaller within each different language they know because they have less experience in each language than monolingual children have in their one language. Early vocabularies in languages other than English have been shown to have an abundance of shape-based nouns (e.g., Colunga and Smith, 2005; Colunga et al., 2009), but the English language itself may especially encourage attention to object features like shape when learning new words. For example, previous research by Cook et al. (2006) found that Japanese adults exhibited a stronger shape bias the longer they stayed in an English-speaking environment; thus a higher fluency in the English language may be indicative of a stronger bias to attend to shape when extending novel words. Thus, the bilingual children in our study may be less shape-biased because they have less experience with naming shape-based objects in either language than monolinguals have in their one language, English. To compensate for this, bilingual children may need to rely more on pragmatic cues in order to help them determine the correct name of an object. If this were the case one would expect to see a similar relationship looking at individual differences among monolingual children. That is, one would expect a difference in the proportion of shape-based nouns in the vocabulary of monolingual children who have a strong shape bias compared to the vocabulary of monolingual children who have a preference to attend more to pragmatic cues when learning novel words.

Another possibility is that bilingual children in general have a weaker shape bias than monolingual children. If this were the case, then it may be easier for pragmatic cues such as eye gaze to override their weak shape bias. Some evidence in the present study suggests that this account is correct. According to Landau et al. (1988), children attend to shape more heavily than any other object feature, such as color or texture. In our study however, bilingual children exhibit less of a shape bias compared to their monolingual peers, which may suggest that their shape bias is not developed as strongly. There could be a variety of reasons for this. One possibility is that bilingual children simply lag in their development of word learning biases. This is consistent with research that shows, for example, that bilingual children do not adhere as strictly to the mutual exclusivity bias - the assumption that categories tend to have a single name - as monolingual children do. Bilingual children are more likely to learn multiple names for the same object (Houston-Price et al., 2010). Indeed, bilingual children have been shown in several task to be more likely to learn multiple names for an object than monolingual children (Au and Glusman, 1990; Davidson et al., 1997; Houston-Price et al., 2010). Whatever the reason, this hypothesized weaker shape bias might in part explain bilingual children's lag in word learning. Is this is the case, one prediction is that bilingual children might show less of a shape bias in situations in which, unlike in our experiment, shape is not highlighted by grouping.

Another possibility to explain why bilingual children use pragmatic gestures to a greater degree is that bilingual children simply learn to attend more to this type of information because they constantly hear two languages and must take notice of who speaks which language (Grassmann and Tomasello, 2010). Moreover, people may be able to communicate successfully in a foreign culture whose language they do not speak by using pragmatic gestures such as pointing and eye gaze. Thus, bilingual children may be more used to attending to pragmatic gestures. It may also be possible that a socio-pragmatic cue such as eye gaze may be more subtle for monolingual children (Jaswal and Hansen, 2006). While monolingual children also pay attention to pragmatic gestures, they may give them less importance because they do not need to attend to different languages spoken around them.

Finally, our results are also consistent with the larger literature on word learning in monolingual children. Research shows that monolingual children pay more attention to object property cues when learning a new word, relative to bilingual children (Rosenblum and Pinker, 1983; Merriman and Kutlesic, 1993; Haryu and Imai, 1999). Parents of monolingual children may be more likely to highlight characteristics of an object aimed to assist the child in distinguishing the referent from nouns that are perceptually similar. For instance, in an example used in Merriman and Kutlesic's (1993), study in teaching the word zebra, a parent may draw attention to its stripes in order to help the child differentiate it from a horse. A bilingual parent may instead tell their child, this is a horse in English, and a caballo in Spanish, thus diminishing the importance given to object properties. However, it must be noted that bilingual children did indeed pay attention to object property 
cues when the experimenter looked toward the objects matching in shape. Thus, our results do not suggest that bilingual children do not use object property cues at all, but that in some instances they are more likely to attend to pragmatics than monolingual children.

This greater reliance on pragmatic cues in bilingual children may result in an enhanced ability to learn words later in life. Results of a study done by Kaushanskaya and Marian (2009) examine the effects of early exposure to two languages on word learning ability. Their results suggest a bilingual advantage, with bilingual adults outperforming monolingual adults in learning novel words. Specifically, bilinguals achieved higher accuracy rates when tested for the meaning of a novel word, and performed superiorly in recall and recognition tasks of the novel word a week after testing. It is possible that bilinguals outperforming monolinguals in their aptitude to learn novel words arises from the relatively higher attention given to pragmatic cues in their early environment. Added attention to these cues may facilitate word learning. In the present study, bilingual participants were able to attend to pragmatic information when the object property cue and pragmatic cue were conflicting, whereas monolingual participants were not. Monolingual participants preserved their attention to highlighted object properties - specifically shape. As suggested previously, bilingual children may be more flexible in attending to linguistic cues, which may heighten their cognitive ability in language acquisition. This early experience with a second language may encourage more attention to pragmatic cues and result in rapid novel word learning.

\section{LIMITATIONS AND FUTURE DIRECTIONS}

The differences in monolingual and bilingual word learning suggest that linguistic environments influence how attention is deployed to different types of linguistically relevant cues, such

\section{REFERENCES}

Akhtar, N., Carpenter, M., and Tomasello, M. (1996). The role of discourse novelty in early word learning. Child Dev. 67, 635-645.

$\mathrm{Au}, \mathrm{T}$., and Glusman, M. (1990). The principle of mutual exclusivity in word learning: to honor or not to honor? Child Dev. 61, 1474-1490.

Baldwin, D. A. (1993). Early referential understanding: infants' ability to recognize referential acts for what they are. Dev. Psychol. 29, 832-843.

Bialystok, E., Barac, R., Blaye, A., and Poulin-Dubois, D. (2010). Word mapping and executive functioning in young monolingual and bilingual children. J. Cogn. Dev. 11, 485-508.

Bialystok, E., and Martin, M. M. (2004). Attention and inhibition in bilingual children: evidence from the dimensional change card sort task. Dev. Sci. 7, 325-339.

Clark, E. V., and Grossman, J. B. (1998). Pragmatic directions and children's word learning. J. Child Lang. 25, $1-18$.

as pragmatic information and object property information. A preference in choosing one cue over the other is likely shaped by the monolingual or bilingual linguistic environment. Followup studies should investigate the degree to which a bilingual child or monolingual child looks at the speaker of a particular word. Furthermore, studies that measure the precise attention children give to these different types of cues, such as on-line measures of eye gaze, instead of comparing behavioral choices, could reveal the definite role that experience plays in shaping children's attention in word learning tasks. Finally, a future study must include a measure to ascertain an equivalency in vocabulary between the two groups of monolingual and bilingual children, as vocabulary may have an effect on a child's readiness to extend a novel word to an object.

\section{CONCLUSION}

This study compared strategies used by monolingual and bilingual children in language acquisition, particularly in situations where cues conflict. In sum, monolingual children may rely less on pragmatic gestures because they hear only one language. On the other hand, bilingual children are simultaneously taught two labels for a word and thus may need pragmatic cues to a greater degree relative to monolinguals in order to learn a word. For example, if a parent is talking about a perro and the child has no clues as to what his or her parent is referring to, he or she may unconsciously follow his or her parent's eye gaze to determine the intended referent. While monolingual children most likely exhibit the same behavior when learning a new word, they do not hear two labels for a word as often as bilingual children. Thus, a monolingual child may utilize a pragmatic cue to learn a word less often than a bilingual child, and vice versa. Overall, these results suggest that learning language in a bilingual environment leads children to attend more to pragmatic cues than a monolingual environment.

examination. Dev. Psychol. 41, 451-463.

Grassmann, S., and Tomasello, M. (2010). Young children follow pointing over words in interpreting acts of reference. Dev. Sci. 13, 252-263.

Haryu, E., and Imai, E. (1999). Controlling the application of the mutual exclusivity assumption in the acquisition of lexical hierarchies. Jpn. Psychol. Res. 41, 21-34.

Haryu, E., and Imai, E. (2002). Reorganizing the lexicon by learning new words: Japanese children's interpretation of the meaning of a new word for a familiar artifact. Child Dev. 73 1378-1391.

Houston-Price, C., Caloghiris, Z., and Raviglione, E. (2010). Language experience shapes the development of the mutual exclusivity bias. Infancy 15, 12-150.

Jaswal, V. K., and Hansen, M. B. (2006). Learning words: children disregard some pragmatic information that conflicts with mutual exclusivity. Dev. Sci. 9, 158-165.
Jones, S. S., and Smith, L. B., Landau, B. (1991). Object properties and knowledge in early lexical learning. Child Dev. 62, 499-516.

Kaushanskaya, M., and Marian, V. (2009). The bilingual advantage in novel word learning. Psychon. Bull. Rev. 16, 705-710.

Landau, B., Smith, L. B., and Jones, S. S. (1988). The importance of shape in early lexical learning. Cogn. Dev. 3, 299-321.

Merriman, W., and Kutlesic, V. (1993). Bilingual and monolingual children's use of two lexical acquisition heuristics. Appl. Psycholinguist. 14, 229-249.

Petitto, L. A., Katarelos, M., Levy, B. G., Gauna, K., Tetreault, K., and Ferraro, V. (2001). Bilingual signed and spoken language acquisition from birth: implications for the mechanisms underlying early bilingual language acquisition. J. Child Lang. 28, 453-496.

Rosenblum, T., and Pinker, S. A. (1983). Word magic revisited: monolingual 
and bilingual children's understanding of the word-object relationship. Child Dev. 54, 773-780.

Samuelson, L.K. (2002). Statistical regularities in vocabulary guide language acquisition in connectionist models and 15-20-month-olds. Dev. Psychol. 38, 1016-1037.

Smith, L. B. (2000). "Learning how to learn words: an associative crane," in Becoming a Word Learner: A Debate on Lexical Acquisition, eds R. M. Golinkoff, K. Hirsh-Pasek, L. Bloom, L. B. Smith, A. M. Woodward, N. Akhtar, M. Tomasello, and G. Hollich (New York: Oxford University Press), 51-80.
Smith, L. B., Colunga, E., and Yoshida, H. (2010). Knowledge as process: contextually-cued attention and early word learning. Cogn. Sci. 34, 1287-1314.

Smith, L. B., Jones, S., Landau, B., Gershkoff-Stowe, L., and Samuelson, L. (2002). Object name learning provides on-the-job training for attention. Psychol. Sci. 13, 13-19.

Soja, N. N., Carey, S., and Spelke, E. S. (1991). Ontological categories guide young children's inductions of word meaning: object terms and substance terms. Cognition 38, 179-211.
Yoshida, H., and Smith, L. B. (2003). Shifting ontological boundaries: how Japanese- and English-speaking children generalize names for animals and artifacts. Dev. Sci. 6, $1-17$.

Conflict of Interest Statement: The authors declare that the research was conducted in the absence of any commercial or financial relationships that could be construed as a potential conflict of interest.

Received: 17 December 2011; accepted: 27 April 2012; published online: 25 May 2012.
Citation: Brojde CL, Ahmed S and Colunga E (2012) Bilingual and monolingual children attend to different cues when learning new words. Front. Psychology 3:155. doi: 10.3389/fpsyg.2012.00155 This article was submitted to Frontiers in Developmental Psychology, a specialty of Frontiers in Psychology.

Copyright (c) 2012 Brojde, Ahmed and Colunga. This is an open-access article distributed under the terms of the Creative Commons Attribution Non Commercial License, which permits noncommercial use, distribution, and reproduction in other forums, provided the original authors and source are credited. 